\title{
OSCILLATIONS DE FONCTIONS ALÉATOIRES GAUSSIENNES À VALEURS VECTORIELLES
}

\author{
X. FERNIQUE
}

\section{Sommaire.}

On étend aux fonctions aléatoires gaussiennes à valeurs vectorielles les résultats de Ito et Nisio sur les oscillations des fonctions aléatoires gaussiennes à valeurs réelles; les oscillations utilisées sont à valeurs vectorielles. Les résultats obtenus permettent d'énoncer une généralisation adaptée de l'alternative de Belayev en mettant pourtant en évidence des phénomènes nouveaux de singularité.

\section{Oscillations de fonctions à valeurs vectorielles, définitions, propriétés générales.}

Soient $(T, \delta)$ un espace pseudométrique, $S$ une partie dense de $T$ et $E$ un espace de Banach; soit de plus $f$ une application de $S$ dans $E$, nous appellerons $(S, \delta)$-oscillation de $f$ et nous noterons $W_{S}(f)$ la fonction sur $T$ à valeurs dans les parties fermées de $E$ définie par

$$
W_{s}(f, t)=\bigcap_{u>0} \overline{\left\{f(s)-f\left(s^{\prime}\right), \delta(t, s)<u, \delta\left(t, s^{\prime}\right)<u,\left(s, s^{\prime}\right) \in S \times S\right\}} .
$$

De même pour tout $t \in T$ et tout $u>0$, nous appellerons $(S, \delta)$-oscillation de $f$ sur la boule $B(t, u)$ de centre $t$ et de rayon $u$ l'ensemble

$$
\text { 1.2. } \quad V_{s}(f, t, u)=\bigcap_{v>0} \overline{\left\{f(s)-f\left(s^{\prime}\right), s \in B(t, u) \cap S, s^{\prime} \in B(t, u) \cap S, \delta\left(s, s^{\prime}\right)<v\right\}} .
$$

On vérifie d'après ces définitions que ces oscillations possèdent les propriétés suivantes:

1.3.1. $V_{s}(f, t, u)$ est une fonction croissante de $u$.

1.3.2. Si $f$ est uniformément continue sur $(S, \delta)$, alors $V_{S}(f, t, u)$ se réduit à $\{0\}$; de plus pour toute autre fonction $g$, on a alors

Reçu le 7 octobre 1985. 


$$
V_{S}(f+g, t, u)=V_{S}(g, t, u) .
$$

1.3.3 $\mathrm{Si} \delta\left(t, t^{\prime}\right)$ est inférieur à $v>0$, alors $V_{S}(f, t, u)$ est contenu dans $V_{s}\left(f, t^{\prime}, u+v\right)$.

1.3.4. $W_{S}(f, t)$ est égal à l'intersection $\bigcap_{u}>{ }_{0} V_{S}(f, t, u)$.

1.3.5. Pour qu'il existe une fonction $g$ sur $T$ à valeurs dans $E$ qui soit continue sur $(T, \delta)$ et coïncide avec $f$ sur $S$, il faut que pour tout $t \in T, W_{S}(f, t)$ se réduise à $\{0\}$; de plus cette condition est suffisante si l'image de $f$ est relativement compacte.

Si $f$ est en fait définie sur $T$, on peut aussi introduire la $(S, \delta)$-demioscillation de $f$, c'est la fonction sur $T$ à valeurs dans les parties fermées de $E$ définie par

$$
U_{S}(f, t)=\bigcap_{u>0} \overline{\{f(s)-f(t), s \in B(t, u) \cap S\}}
$$

on vérifie que $W_{S}(f, t)$ contient l'ensemble des différences des couples d'éléments de $U_{S}(f, t)$; il y a en fait égalité si la trajectoire de $f$ sur $S$ est relativement compacte dans $E$. Sous une telle hypothèse, la notion d'oscillation devient beaucoup plus maniable, on a par exemple:

Proposition 1.5. On suppose que la trajectoire de $f$ sur $S$ est contenue dans une partie compacte $K$ de $E$; dans ces conditions, pour tout $u>0$, il existe une famille finie $\left(y_{1}, \ldots, y_{n}\right)$ de formes linéaires continues sur $E$ ne dépendant que de $K$ et de u telle que pour tout $t \in T$, on ait

$$
\left.\sup \left\{|x|, x \in W_{S}\left(\left\langle f, y_{k}\right\rangle, t\right), k \in[1, n]\right\} \leqq 1 \Rightarrow \sup \left\{\|x\|, x \in W_{S} f, t\right)\right\} \leqq u .
$$

En effet puisque $K$ est compact, la topologie de $E$ et la topologie affaiblie $y$ coïncident; il existe donc une famille finie $\left(y_{1}, \ldots, y_{n}\right)$ de formes linéaires continues sur $E$ telle que pour tout couple $\left(x, x^{\prime}\right)$ d'éléments de $K$, on ait

$$
\sup \left\{\left|\left\langle x-x^{\prime}, y_{k}\right\rangle\right|, 1 \leqq k \leqq n\right\} \leqq 1 \Rightarrow\left\|x-x^{\prime}\right\| \leqq u,
$$

et on constate que cette famille vérifie les propriétés énoncées.

\section{Oscillations de fonctions aléatoires gaussiennes à valeurs vectorielles.}

Dans ce paragraphe, $X$ désigne une fonction aléatoire gaussienne centrée (f.a.g.) sur un espace pseudométrique séparable $(T, \delta)$ à valeurs dans un espace 
de Banach séparable $E$; $E^{\prime}$ est le dual topologique de $E$ et $E_{1}^{\prime}$ en est la boule unité; on note $\hat{d}$ et $D$ les pseudométriques sur $T$ définies par

$$
\hat{d}^{2}(s, t)=\sup \left\{E\langle X(s)-X(t), y\rangle^{2}, y \in E_{1}^{\prime}\right\}, \quad D^{2}(s, t)=E\|X(s)-X(t)\|^{2},
$$

enfin $S$ est une partie dénombrable et dense de $T$. On suppose dans tout ce paragraphe que l'application canonique de $(T, \delta)$ dans $(T, \hat{d})$ est uniformément continue. On se propose d'étudier les propriétés des oscillations $\left\{W_{S}(X(\omega), t), \omega \in \Omega, t \in T\right\}$. Les résultats principaux sont contenus dans lesdeux théorèmes suivants:

THÉORÈME 2.1. La $(S, \delta)$-oscillation de $X$ est non aléatoire au sens suivant: il existe une partie négligeable $N_{S}$ de $\Omega$ et une application $w_{S}$ de $T$ dans l'ensemble des parties fermées de E telles que

$$
\forall \omega \notin N_{S}, \forall t \in T, \quad W_{S}(X(\omega), t)=w_{S}(t) .
$$

Si de plus l'application canonique de $(T, \delta)$ dans $(T, D)$ est continue, alors la fonction $w_{S}$ est indépendante de $S$, c'est-à-dire qu'il existe une application $w$ de $T$ dans l'ensemble des parties fermées de $E$ et pour tout partie $S$ dénombrable dense de $T$, il existe une partie négligeable $N_{S}$ de $\Omega$ telles que

$$
\forall \omega \notin N_{S}, \forall t \in T, \quad W_{S}(X(\omega), t)=w(t) .
$$

THÉORÈME 2.2. La $(S, \delta)$-demi-oscillation de $X$ est non aléatoire au sens suivant: il existe une application $u_{S}$ de $T$ dans l'ensemble des parties fermées de $E$ et pour tout élément $t$ de $T$ une partie négligeable $N_{S}(t)$ de $\Omega$ telles que

$$
\forall t \in T, \forall \omega \notin N_{S}(t), \quad U_{S}(X(\omega), t)=u_{S}(t) .
$$

Si de plus l'application canonique de $(T, \delta)$ dans $(T, D)$ est continue, alors $u_{s}$ est indépendante de $S$.

Nous ne démontrerons que le Théorème 2.1., la preuve du Théorème 2.2. n'apportant aucun élément supplémentaire. Le schéma de preuve sera très semblable à celui utilisé dans le cas réel par Ito et Nisio [5] sous la forme développée dans [2]. L'idée fondamentale est d'utiliser un développement de Karhunen-Loeve de $X$ pour montrer que les ensembles $V_{S}(X(\omega), t, u)$ sont p.s. non aléatoires; nous développerons donc d'abord les notions nécessaires sur les espaces autoreproduisants asociés aux f.a.g. à valeurs vectorielles; nous résoudrons aussi les difficultés liées à la measurabilité de l'application $\omega \rightarrow V_{S}(X(\omega), t, u)$ en utilisant pour cela une technique efficace dans l'étude de 
la loi vectorielle du logarithme itéré (où l'ensemble limite est effectivement une $(\mathbf{N}, \delta)$-demi-oscillation au point à l'infini pour une fonction aléatoire sur $\overline{\mathbf{N}}$ à valeurs vectorielles).

\subsection{NOTIONS SUR LES ESPACES AUTOREPRODUISANTS.}

2.3.1. A la f.a.g. $X$ à valeurs vectorielles, est associé la f.a.g. $\tilde{X}$ sur $T \times E$ à valeurs réelles définie par

$$
\forall t \in T, \forall y \in E^{\prime}, \quad\langle X(t), y\rangle=\tilde{X}(t, y) ;
$$

à fout élément $\tilde{h}$ de l'espace autoreproduisant $\widetilde{H}$ lié à $\tilde{X}$ est associé un élément $k$ de $L^{2}(\Omega, \mathscr{F}, P)$ et on a

$$
\forall t \in T, \forall y \in E^{\prime}, \quad \tilde{h}(t, y)=\int k \tilde{X}(t, y) d P .
$$

Ceci implique que pour tout $t \in T$, l'application $y \rightarrow \widetilde{h}(t, y)$ est définie par l'élément $h(t)=\int k X(t) d P$ de l'espace autoreproduisant $H(t)$ lié à $X(t)$; puisque $X(t)$ est un vecteur gaussien à valeurs dans $E, h(t)$ est un élément de $E$ et on a

$$
\forall t \in T, \forall y \in E^{\prime}, \quad\langle h(t), y\rangle=\tilde{h}(t, y)
$$

il en résulte en particulier que pour tout couple $(s, t)$ d'éléments de $T$ et tout élément $y$ de $E_{1}^{\prime}$, on a

$$
\langle h(s)-h(t), y\rangle=\int k\langle X(s)-X(t), y\rangle d P \leqq\|k\|_{L^{2}}\left(E\langle X(s)-X(t), y\rangle^{2}\right)^{1 / 2},
$$

et donc

$$
\|h(s)-h(t)\|_{E} \leqq\|\tilde{h}\|_{\hat{H}} \hat{d}(s, t)
$$

ceci signifie que l'application $h$ de $T$ dans $E$ est uniformément continue sur $(T, \hat{d})$ et vu nos hypothèses, sur $(T, \delta)$.

2.3.2. Puisque $(T, \delta) \times\left(E_{1}^{\prime}, w\right)$ est séparable, $\tilde{H}$ l'est aussi. Soit $\left(\tilde{h}_{n}\right)$ une base orthonormale de $\tilde{H}$; il existe alors une suite gaussienne normale $\left(\lambda_{n}\right)$ telle que

$$
\forall t \in T, \forall y \in E^{\prime}, \quad \tilde{X}(t, y)=\sum \lambda_{n} \tilde{h}_{n}(t, y) \text { p.s. }
$$

pour tout $t \in T, \sum \lambda_{n} h_{n}(t)$ est donc une série de variables aléatoires indépen- 
dantes à valeurs dans $E$ telle que

$$
\forall y \in E^{\prime}, \quad P\left\{\langle X(t), y\rangle=\sum \lambda_{n}\left\langle h_{n}(t), y\right\rangle\right\}=1 ;
$$

bien que les $h_{n}(t)$ ne soient pas nécessairement orthonormaux dans $H(t)$ (si les $\lambda_{n}$ ne sont pas $X(t)$-mesurables), ceci suffit pour que pour tout $t \in T$, la série $\sum \lambda_{n} h_{n}(t)$ converge p.s. dans $E$ vers $X(t)$. Nous notons $S_{n}(t)$ et $R_{n}(t)$ la somme partielle et le reste de rang $n$ de cette dernière série; il existe une partie négligeable $N$ telle que

$$
\forall n \in \mathbf{N}, \forall \omega \notin N, \forall t \in S, \quad X(\omega, t)-S_{n}(\omega, t)=R_{n}(\omega, t) ;
$$

comme pour tout $\omega \in \Omega, S_{n}(\omega)$ est d'après 2.3.1. uniformément continue sur $(T, \delta)$, la relation ci-dessus et la propriété 1.3.2. permettent de calculer pour tout $n \in \mathbf{N}$, les oscillations de $X(\omega)$ en fonction de celles de $R_{n}(\omega)$.

2.4. Sur la mesurabilité des oscillations des f.a.g.

Lemme 2.4.1. Pour tout $t \in T$, tout $u>0$ et tout fermé $F$ de E, l'ensemble

$$
\Omega(X, F)=\left\{\omega \in \Omega: F \subset V_{S}(X(\omega), t, u)\right\}
$$

est mesurable pour la tribu engendrée par $X$; sa probabilité vaut zéro ou un.

Démonstration. Pour tout élément $x$ de $E, \Omega(X, x)$ est l'ensemble $\{\omega \in \Omega$ : $\left.\left.x \in V_{S}(X(\omega)), t, u\right)\right\}$ et la définition de $V_{S}$ montre puisque $S$ est dénombrable que cet ensemble est mesurable pour la tribu engendrée par $X$; le résultat 2.3.1. montre que pour tout entier $n, \Omega(X, x)$ est p.s. égal à $\Omega\left(R_{n}, x\right)$ mesurable pour la tribu engendrée par $R_{n}$; sa probabilité vaut donc zéro ou un. Soient alors $F$ une partie fermée de $E$ et $\left(x_{n}\right)$ une suite dense dans $F, \Omega(X, F)$ est égal à l'intersection des $\Omega\left(X, x_{n}\right)$ et le résultat s'ensuit.

Lemme 2.4.2. Supposons l'espace d'épreuves complet; pour tout $t \in T$, tout $u>0$ et toute partie ouverte $U$ de E, l'ensemble

$$
\Omega(U)=\left\{\omega \in \Omega: U \cap V_{S}(X(\omega), t, u) \neq \varnothing\right\}
$$

est mesurable; sa probabilité vaut zéro ou un.

Demonstration. Soit $U$ une partie ouverte de $E$; si $\Omega(U)$ est négligeable, la conclusion du lemme est vérifiée pour $U$; nous supposons donc dans la suite de la preuve que la probabilité extérieure de $\Omega(U)$ est non nulle et nous allons construire une partie mesurable $A$ de $\Omega$ telle que 


$$
A \subset \Omega(U), \quad P(A)=1,
$$

et ceci établira la conclusion du lemme dans tous les cas.

Notons d'abord que puisque $E$ est séparable, pour tout ouvert $G$ de $E$ et tout entier $p>0$, il existe un recouvrement dénombrable $\left(G_{k}(G, p), k \in \mathbf{N}\right)$ de $G$ par des parties ouvertes de diamètre inférieur à $1 / p$ dont les adhérences sont contenues dans $G$; si la probabilité extérieure de $\Omega(G)$ est non nulle, il existe au moins un élément $k$ de $\mathbf{N}$ tel que la probabilité extérieure de $\Omega\left(G_{k}(G, p)\right)$ soit aussi non nulle.

Dans ces conditions, si $P^{*}(\Omega(U))$ est non nulle, on construit par récurrence une suite $\left(U_{p}\right)$ de parties ouvertes de $E$ telles que

$$
U_{0}=U ; \quad \forall p>0, U_{p} \in\left(G_{k}(p-1, p), k \in \mathbf{N}\right) ; \quad \forall p \geqq 0, P^{*} \Omega\left(U_{p}\right)>0 .
$$

La suite $\left(U_{p}, p \geqq 0\right)$ engendre alors un filtre de Cauchy convergeant dans l'espace complet $E$ vers un élément $x$ appartenant à $\bar{U}_{1}$ donc à $U$ et on a

$$
\forall p>0, \quad U_{p} \subset B(x, 1 / p)
$$

Par ailleurs pour tout entier $p>0$, l'ensemble $\Omega\left(U_{p}\right)$ est contenu dans l'ensemble

$$
\begin{aligned}
\Omega^{\prime}(p, x)= & \left\{\forall v>0, \exists s \in B(t, u) \cap S, \exists s^{\prime} \in B(t, u) \cap S:\right. \\
& \left.\delta\left(s, s^{\prime}\right)<v,\left\|X(s)-X\left(s^{\prime}\right)-x\right\| \leqq v+1 / p\right\}
\end{aligned}
$$

mesurable pour la tribu engendrée par $X$ et comme précédemment pour la tribu complète engendrée par $R_{n}$; puisque $P^{*} \Omega\left(U_{p}\right)$ est non nulle, $P \Omega^{\prime}(p, x)$ est aussi non nulle, donc égale à 1 ; on note $A$ l'ensemble $\cap_{p>0} \Omega^{\prime}(p, x)$, il est de probabilité un et pour tout élément $\omega$ de $A$, on a

$$
\begin{aligned}
\forall p>0, & \exists s \in B(t, u) \cap S, \\
& \exists s^{\prime} \in B(t, u) \cap S: \delta\left(s, s^{\prime}\right)<1 / p,\left\|X(s)-X\left(s^{\prime}\right)-x\right\|<2 / p ;
\end{aligned}
$$

ceci signifie que $A$ est contenu dans $\Omega(x)$ et donc dans $\Omega(U)$; on a mis en évidence un ensemble mesurable de probabilité un contenu dans $\Omega(U)$; le lemme est démontré.

2.5. Démonstration du Théorème 2.1. Pour tout $t \in T$ et tout $u>0$, nous notons $v(t, u)$ l'ensemble $\{x: P \Omega(X, x)=1\}$; on constate que $v(t, u)$ est fermé et que $P \Omega(X, v(t, u))$ est égale à 1 ; soit par ailleurs $U$ le complémentaire ouvert de $v(t, u)$, alors pour tout $x \in U, P \Omega(X, x)$ est nulle et le Lemme 2.4.2. montre 
que la probabilité extérieure de $\Omega(U)$ est nulle. Il existe donc une partie négligeable $N(t, u)$ de $\Omega$ telle que

$$
\forall \omega \notin N(t, u), \quad v(t, u) \subset V_{S}(X(\omega), t, u), \quad[v(t, u)] \cap V_{S}(X(\omega), t, u)=\varnothing,
$$

c'est-à-dire

2.5.1.

$$
\forall \omega \notin N(t, u), \quad v(t, u)=V_{S}(X(\omega), t, u) .
$$

Les propriétés des oscillations montrent que la fonction $v$ vérifie comme elles :

2.5.2. $v(t, u)$ est une fonction croissante de $u$.

2.5.3. Si $\delta\left(t, t^{\prime}\right)$ est inférieur à $u^{\prime}>0$, alors $v(t, u)$ est contenu dans $v\left(t^{\prime}, u+u^{\prime}\right)$. On note $N_{S}$ la réunion $\bigcup_{t \in S} \bigcup_{u \in Q^{+}} N(t, u)$, c'est une partie négligeable; on pose aussi $w_{S}(t)$ égal à l'intersection $\bigcap_{u>0} v(t, u)$, on va montrer que $N_{s}$ et $w_{S}$ ainsi construits vérifient la conclusion 2.1.1. du théorème.

En effet pour tout $t \in T$ et tout couple $\left(u, u^{\prime}\right)$ de nombres $>0$, puisque $S$ est dense dans $T$, il existe un élément $s$ de $S$ et un nombre $u^{\prime \prime}$ tels que

$$
\delta(s, t)<u^{\prime \prime}<u^{\prime} / 2, \quad u+u^{\prime \prime} \in \mathbf{Q}^{+}
$$

d'après les propriétés 1 3.3., 2.5.1. et 2.5.2., pour tout $\omega \notin N_{S}$, on aura alors

$$
V_{S}(X,(\omega), t, u) \subset V_{S}\left(X(\omega), s, u+u^{\prime \prime}\right)=v\left(s, u+u^{\prime \prime}\right) \subset v\left(t, u+u^{\prime}\right),
$$

et aussi de la même manière

$$
v(t, u) \subset v\left(s, u+u^{\prime \prime}\right)=V_{S}\left(X(\omega), u+u^{\prime \prime}\right) \subset V_{S}\left(X(\omega), t, u+u^{\prime}\right) ;
$$

il en résulte successivement

$$
\begin{aligned}
W_{S}(X(\omega), t) & =\bigcap_{u>0} V_{S}(X(\omega), t, u) \subset \bigcap_{u>0} v(t, 2 u)=w_{S}(t), \\
w_{S}(t) & =\bigcap_{u>0} v(t, u) \subset \bigcap_{u>0} V_{S}(X(\omega), t, u)=W_{S}(X(\omega), t),
\end{aligned}
$$

et ces deux relations établissent la propriété 2.1.1.

Supposons maintenant l'application $(T, \delta) \rightarrow(T, D)$ continue, notons $S_{1}, S_{2}$ deux parties dénombrables denses de $T, N_{1}$ et $N_{2}$ les parties négligeables, 
$w_{1}$ et $w_{2}$ les oscillations non aléatoires qui leur sont associées par la propriété 2.1.1. Pour tout élément $s$ de $S_{1}$, il existe une suite $\left(s_{n}(s)\right)$ extraite de $S_{2}$ convergeant vers $s$ et telle que $X\left(s_{n}(s)\right)$ converge p.s. vers $X(s)$; il existe donc une partie négligeable $N_{3}$ de $\Omega$ telle que

$$
\forall \omega \notin N_{3}, \forall s \in S_{1}, \quad X(\omega, s)=\lim X\left(\omega, s_{n}(s)\right) .
$$

Soient alors $\omega \notin N_{1} \cup N_{2} \cup N_{3}, t \in T, x \in w_{1}(t)$ et $u>0$; la définition de $w_{1}(t)$ montre qu'il existe des éléments $s$ et $s^{\prime}$ de $S_{1}$ tels que

$$
\left\|X(\omega, s)-X\left(\omega, s^{\prime}\right)-x\right\| \leqq u / 3, \quad \delta(t, s) \leqq u / 2, \quad \delta\left(t, s^{\prime}\right) \leqq u / 2 ;
$$

il existe aussi un entier $n>0$ tel que

$$
\begin{aligned}
& \left\|X(\omega, s)-X\left(\omega, s_{n}(s)\right)\right\| \leqq u / 3, \quad \delta\left(s, s_{n}(s)\right) \leqq u / 2 \\
& \| X\left(\omega, s^{\prime}\right)-X\left(\omega, s_{n}\left(s^{\prime}\right) \| \leqq u / 3, \quad \delta\left(s^{\prime}, s_{n}\left(s^{\prime}\right)\right) \leqq u / 2\right.
\end{aligned}
$$

on aura alors aussi

$$
\left\|X\left(\omega, s_{n}(s)\right)-X\left(\omega, s_{n}\left(s^{\prime}\right)\right)-x\right\| \leqq u, \quad s_{n}(s) \in B(t, u) \cap S_{2}, s_{n}\left(s^{\prime}\right) \in B(t, u) \cap S_{2},
$$

et ceci montre que $x$ appartient aussi à $w_{2}(t)$, établit l'inclusion $w_{1}(t) \subset w_{2}(t)$ et donc l'égalité; le théorème est démontré.

2.6. Remarques sur le champ d’application des théorèmes. Si $(T, \delta)$ est quasicompact, si $X$ est tendu, c'est-à-dire si pour tout $u>0$, il existe une partie compacte $K$ de $E$ telle que pour tout $t \in T, P\{X(t) \notin K\}$ soit inférieure à $u$, si de plus $X$ est faiblement continue en probabilité, c'est-à-dire si pour tout élément $y$ de $E^{\prime}$, la fonction aléatoire réelle $t \rightarrow\langle X(t), y\rangle$ est continue en probabilité, on sait [3] que les injections $(T, \delta) \rightarrow(T, D) \rightarrow(T, \hat{d})$ sont uniformément continues; dans ces conditions les hypothèses des deux théorèmes sont alors vérifiées. Ce sera en particular le cas si $X$ est la restriction à une partie compacte $T$ d'une f.a.g. sur $\mathbf{R}$ ou $\mathbf{R}^{n}$ stationnaire faiblement continue en probabilité.

\section{Application à l'étude des trajectoires des f.a.g. stationnaires ou à accrois- sements stationnaires et à valeurs vectorielles.}

Dans ce paragraphe, $(G, \delta)$ est un groupe métrique localement compact séparable, $T$ en est une partie compacte d'intérieur $\stackrel{+}{T}$ que l'on suppose dense dans $T ; S$ est une partie dénombrable de $\stackrel{T}{T}$ dense dans $T ; E$ est un espace de Banach séparable; $X$ est une f.a.g. sur $G$, stationnaire ou à accrois- 
sements stationnaires, à valeurs dans $E$ et on suppose qu'elle est faiblement continue en probabilité.

Si $E=\mathbf{R}$, alors l'alternative de Belayev montre [1] que, soit $X$ a p.s. des trajectoires non bornées sur $S$, soit il existe une f.a.g. $X^{\prime}$ équivalente à $X$ et ayant ses trajectoires continues sur $G$. On se propose ici d'etudier les extensions de cette alternative aux espaces de Banach généraux. Ce ne'st pas possible dans les termes ci-dessus comme le montre l'example suivant [3]:

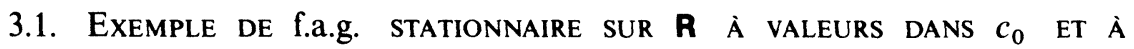
TRAJECTOIRES SINGULIÈreS. Soient $\left(\lambda_{n}\right)$ une suite gaussienne normale et $U$ une f.a.g. stationnaire sur $\mathbf{R}$ normalisée à valeurs réelles ayant p.s. des trajectoires continues. On suppose que

$$
\lim _{t \rightarrow \infty} E(U(0) U(t))=0
$$

de sorte que

$$
\begin{aligned}
& E \sup \{U(t) / \sqrt{2 \log (2+t)}, t \geqq 0\}<\infty, \\
& \quad \lim _{T \rightarrow \infty} \sup \{U(t) / \sqrt{2 \log (2+T)}, 0 \leqq t \leqq T\}=1, \text { p.s. } ;
\end{aligned}
$$

soit de plus $\left(U_{n}\right)$ une suite de copies de $U$ indépendantes; pour tout $t \in \mathbf{R}$, on pose

$$
X(t)=\left\{U_{n}\left(t \exp \left(n^{2} / 2\right)\right) /(n+1), n \in \mathbf{N}\right\} .
$$

Dans ces conditions, la suite $\left(X_{n}(t)\right)$ a même loi que la suite $\left(\lambda_{n} /(n+1)\right)$ de sorte que $X(t)$ est p.s. un élément de l'espace $c_{0}$; ceci signifie que $X=\{X(t), t \in \mathbf{R}\}$ est une f.a.g. à valeurs dans $c_{0}$. Par ailleurs pour tout élément $h$ de $\mathbf{R}$, la loi de la translatée $\tau_{h} X$ est déterminée par l'ensemble des

$$
E\left(X_{n}(t+h) X_{m}(s+h)\right), \quad(s, t) \in \mathbf{R} \times \mathbf{R},(n, m) \in \mathbf{N} \times \mathbf{N}
$$

si $n \neq m$, cette espérance est nulle par l'indépendance, indépendamment de $h$; si $n=m$, cette espérance est aussi indépendamment de $h, E\left(X_{n}(t) X_{n}(s)\right)$ puisque $U_{n}$ est stationnaire: ceci montre que $X$ est une f.a.g. stationnaire. On a de plus $([3$, Théorème 1.2.])

$$
\begin{aligned}
E \sup _{n \in \mathbb{N}} \sup _{t \in[0,1]}\left|X_{n}(t)\right| \leqq & \sup _{n \in \mathbb{N}} E \sup _{t \in[0,1]} \frac{U\left(t \exp \left(n^{2} / 2\right)\right)}{n+1}+ \\
& +(\pi / 2) E \sup _{n \in \mathbb{N}}\left(\lambda_{n} /(n+1)\right) ;
\end{aligned}
$$


les deux termes du second membre sont bornés si bien que $X$ a p.s. des trajectoires sur $[0,1]$ bornées dans $c_{0}$. Par contre, fixtant $u \in[0,1]$, on a pour tout $T \geqq 1$, en choisissant $n$ égal à la partie entière de $\sqrt{2 \log (T / u)}+1$

$$
E \sup _{\substack{0 \leqq t \leqq u \\ t \in \mathbf{Q}}}\|X(0)-X(t)\|_{c_{0}} \geqq E \sup _{\substack{0 \leqq t \leqq u \\ t \in \mathbf{Q}}}\left|X_{n}(0)-X_{n}(t)\right| \geqq E \sup _{0 \leqq t \leqq T} \frac{|U(0)-U(t)|}{1+\sqrt{2 \log (T / u)}}
$$

de sorte que pour tout $u \in[0,1]$, faisant tendre $T$ vers l'infini, on obtient

$$
E \sup \left\{\|X(0)-X(t)\|_{c_{0}}, 0 \leqq t \leqq u, t \in \mathbf{Q}\right\} \geqq 1 .
$$

Pour toute f.a.g. $X^{\prime}$ équivalente à $X$, on aura donc:

$$
\lim _{\substack{t \rightarrow 0 \\ t \in \mathbf{Q}}} \sup \left\|X^{\prime}(0)-X^{\prime}(t)\right\|_{c_{0}} \geqq 1 \quad \text { p.s. }
$$

et les trajectoires de $X^{\prime}$ seront p.s. discontinues à l'origine.

On a pourtant dans tout espace de Banach séparable l'extension suivante de l'alternative de Belayev:

THÉORÈME 3.2. Soit $X$ une f.a.g. stationnaire ou à accroissements stationnaires sur le groupe $G$ à valeurs dans $E$ et faiblement continue en probabilité; on a alors l'alternative suivante:

ou bien il existe une f.a.g. $X^{\prime}$ équivalente à $X$ ayant p.s. des trajectoires continues, ou bien toute f.a.g. $X^{\prime}$ équivalente à $X$ a p.s. sur $T$ des trajectoires qui ne sont pas relativement compactes.

Dans la démonstration du théorème, on utilisera le lemme suivant:

Lemme 3.2.1. Soit $X$ une f.a. sur un ensemble dénombrable $S$ à valeurs dans un espace de Banach séparable E. On note A l'ensemble

$$
\{\omega \in \Omega:\{X(\omega, t), t \in S\} \text { est relativement compact dans } E\} \text {; }
$$

alors $A$ est mesurable; si $X$ est gaussien, alors $P(A)$ vaut zéro ou un; si de plus $P(A)$ est non nul, il existe un compact $K$ de $E$ tel que

$$
\forall a>0, \quad P\{\forall t \in S, X(t) \in K / a\}>1-a .
$$

Démonstration du lemme. Soit $\left(x_{n}\right)$ une suite dense dans $E$; puisque $E$ est complet, les ensembles relativement compacts $y$ sont les ensembles pré- 
compacts; dans ces conditions,

$$
A=\bigcap_{n \in \mathbb{N}} \bigcup_{m \in \mathbf{N}} \bigcap_{t \in S}\left\{X(t) \in \bigcup_{j=1}^{m} B\left(x_{j}, 4^{-n}\right)\right\}
$$

ceci montre que $A$ est mesurable; supposons maintenant $P(A)>p>0$ et $X$ gaussien, il existe donc une application $m$ de $\mathbf{N}$ dans $\mathbf{N}$ telle que

$$
\forall n \in \mathbf{N}, \quad P \bigcap_{t \in S}\left\{X(t) \in \bigcup_{m=1}^{m(n)} B\left(x_{j}, 4^{-n}\right)\right\}>p ;
$$

notons $C_{n}$ l'enveloppe symétrique convexe de $\left(x_{m}, m \in[1, m(n)]\right)$, alors $C_{n}$ est compacte, convexe, symétrique et

$$
\forall n \in \mathbf{N}, \quad P\left\{(X(t), t \in S) \in\left[C_{n}+B\left(0,4^{-n}\right)\right]^{S}\right\}>p
$$

dans $E^{S},(X(t), t \in S)$ est gaussien et $\left[C_{n}+B\left(0,4^{-n}\right)\right]^{S}$ est symétrique convexe, on a donc d'après les propriétés d'intégrabilité des vecteurs gaussiens (voir $[4,0.3 .4]$.

$$
\forall a>0, \forall n \in \mathbf{N}, \quad P\left\{\forall t \in S, X(t) \in 2^{n+3}\left(C_{n}+B\left(0,4^{-n}\right)\right) /(p a)\right\}>1-a 2^{-(n+1)},
$$

et par suite

$$
P\left\{\forall t \in S,\left(X(t) \in \bigcap_{n \in \mathbb{N}} 2^{n+3}\left(C_{n}+B\left(0,4^{-n}\right)\right) /(p a)\right\}>1-a ;\right.
$$

dans $E$, l'ensemble

$$
K=\bigcap_{n \in \mathbb{N}} \overline{\left\{2^{n+3} C_{n} / p+B\left(0,2^{-n+3} / p\right)\right\}}
$$

est précompact et fermé donc compact, on a

$$
P\{\forall t \in S, X(t) \in K / a\} \geqq(1-a),
$$

et en particulier

$$
P(A) \geqq P\{\exists a>0: \forall t \in S, X(t) \in K / a\}=1
$$

de sorte que le lemme est démontré. 
3.3. Démonstration du Théorème 3.2. Pour démontrer ce théorème, il suffit d'établir que dans ses conditions, s'il existe une f.a.g. $X^{\prime}$ équivalente à $X$ telle que

$$
P^{*}\left\{\omega \in \Omega:\left\{X^{\prime}(\omega, t), t \in T\right\} \text { est relativement compact }\right\}>0,
$$

alors il existe une f.a.g. $X^{\prime \prime}$ sur $T$ coïncidant avec $X^{\prime}$ sur $S$ et ayant p.s. des trajectoires continues sur $T$; il suffit donc (propriété 1.3.5.) de montrer que sous l'hypothèse indiquée, l'oscillation $w_{S}$ associée à $X$ est nulle sur $T$ et que l'image de $S$ par $X$ est relativement compacte. Or cette hypothèse implique (Lemme 3.2.1.) qu'il existe un compact $K$ de $E$ tel que

$$
\begin{aligned}
& P\left\{\exists a \in \mathbf{R}^{+}: \forall t \in S, X(t) \in a K\right\}=1, \\
& P\{\forall t \in S, X(t) \in K\} \geqq \frac{1}{2} .
\end{aligned}
$$

Pour tout élément $y$ de $E^{\prime}$, les trajectoires de $\langle X, y\rangle$ sur $S$ sont alors bornées avec probabilité supérieure à $\frac{1}{2}$; l'alternative classique de Belayev montre que p.s. les mêmes trajectoires sont aussi uniformément continues sur $S$ de sorte que

$$
\forall y \in E^{\prime}, \quad P\left\{\forall t \in T, W_{S}(\langle X(\omega), y\rangle, t)=0\right\}=1 ;
$$

la Proposition 1.5. montre donc

$$
P\left\{\forall t \in T, W_{S}(X(\omega), t)=\{0\}\right\} \geqq P\{\forall t \in S, X(t) \in K\} \geqq \frac{1}{2} ;
$$

le Théorème 2.1. implique alors

$$
P\left\{\forall t \in T, W_{S}(X(\omega), t)=\{0\}\right\}=1
$$

et finalement la conclusion du théorème.

\section{RÉFÉRENCES}

1. Yu. K. Belyaev, Continuity and Hölder's conditions for sample functions of stationary Gaussian processes, (Proc. 4th Berkeley Sympos. Math. Statist. and Prob.), Vol. 2, pp. 23-33. Univ. California Press, Berkeley, California, 1961.

2. X. Fernique, Régularité des trajectoires des fonctions aléatoires Gaussiennes, (École d'Eté de Prob. de Saint-Flour IV, 1974), ed. P.-L. Hennequin, (Lecture Notès i Math. 480), pp. 1-96. Springer-Verlag, Berlin-Heidelberg-New York, 1975.

3. X. Fernique, Fonctions aléatoires gaussiennes à valeurs vectorielles, á paraître.

4. X. Fernique, Les vecteurs aléatoires gaussiennes et leurs espaces autoreproduisants, Technical Report Series in Statist. and Probab., Ottawa Univ., 34, 1985. 
5. M. Nisio et K. Ito, On the oscillation functions of Gaussian processes, Math. Scand. 22 (1968), 209-223.

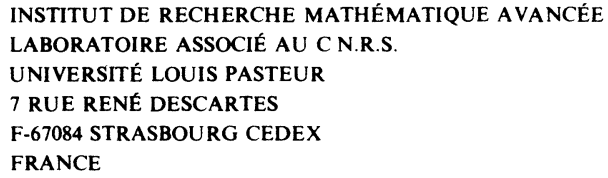

